\title{
Stereotypen, Feindbilder und die Rolle der Medien
}

\author{
von Michael Schmolke
}

„Mit den Rumänen ist es so: Der Ceausescu hat genau gewußt, warum er sie in sein Land gesperrt hat. Ein Rumäne ist nämlich von Natur aus ein Parasit. Seine innere Stimme gebietet ihm, sich unter fremde Völker zu mischen und auf diese Weise das Rumänenuntermenschentum über die ganze Welt zu verbreiten. Deswegen ist es richtig, daß wir ihnen Hilfsgüter schicken, aber es wäre falsch, sie nach Österreich kommen zu lassen, sonst könnte man bald die Österreicher nicht mehr von den Rumänen unterscheiden."

Das stammt nicht von mir, sondern, wie die Leser der "Wochenpresse“ wissen, von dem bekannten Wiener Kabarettisten Georg Kreisler ${ }^{1}$, ist also ironisch-satirisch zu verstehen. Wenden wir uns der nicht-satirischen Wirklichkeit zu! Im deutschen Sprachgebiet kennt man die Redewendung vom "preußischen Charme“ und der „österreichischen Präzision“.

Aber: Welche Eigenschaften setzen die Österreicher nun wirklich auf die ersten vier Plätze, wenn sie aufgefordert werden, ihre Vorzüge selbst zu beurteilen?

\section{Pünktlichkeit \\ 2. Freundlichkeit \\ 3. Fleiß \\ 4. Genauigkeit.}

Das ist nicht von Georg Kreisler. Wir kennen diese Rangliste, weil IMAS sie im November 1989 per Umfrage ermittelt hat ${ }^{2}$. Woher wußten die Befragten, woher nahmen sie, was sie dann zur Antwort gegeben haben? Oder: Woher wissen die Spötter, daß Charme nicht gerade eine Eigenschaft der Preußen ist, und Präzision nicht doch die Stärke der Österreicher?

Kulturpessimisten werden jetzt schnell mit der Meinung präsent sein, daß die Menschen solche Urteile aus den Medien haben. Weit gefehlt. Längst bevor es die modernen Medien gab, hatten die Menschen Bilder von sich und von den anderen, Selbstbilder, Freundbilder, Feindbilder. Selbst der liebe Gott wurde, obwohl er als gebote-gebender Gott dem Bildermachen kritisch gegenüberstand, klischiert: der Vater mit weißem Rauschebart, der Sohn als milder Langhaariger mit Sandalen.

Feindbilderkataloge gab es schon sehr früh, und was in ihnen dem Türken oder dem Mohren zugeschrieben wurde, ist gar nicht weit weg von dem, was heute über Türken oder Schwarze gesagt wird. Solche Kataloge sind zwar

Prof. Dr. Michael Schmolke, Mitherausgeber von CS, ist Leiter des Instituts für Publizistikund Kommunikationswissenschaft der Universität Salzburg. 
schon gedruckt, also als Medien, auf uns gekommen, aber doch aus so früher Zeit, daß man fragen muß: Woher kamen ihre Inhalte? Und wer solche Produkte unters Volk brachte, woher wußte er, daß er Anklang finden würde mit einem illustrierten Katalog von Eigenschaften europäischer Völker, in dem es z. B. von den Polen heißt, sie seien bäuerisch, achteten den Verstand gering, sie seien mittelmäßig, prasserisch, ungestüm, zänkisch, sie trügen sich langröckig und ihr Leben ende im Stall ${ }^{3}$.

Die Bilder sind älter als ihre Vermittlung durch Medien. Wir nennen sie Stereotypen: harte feste Gepräge. Bilder also, die sich nicht leicht verändern lassen. Stereotypen sind so unscharf wie nützlich. Sie helfen uns das komplizierte und täglich komplizierter werdende Leben bestehen. Feindbilder sind ein Sonderfall von Stereotypen, jener Sonderfall, in dem das feste Bild so negativ besetzt ist, daß es in uns Abwehrbereitschaft, Feindseligkeit, Aggressionen auslöst.

Was die Medien angeht, so beobachten wir eine scheinbar paradoxe Entwicklung: Als sie in ihren Anfängen - damals nur als Gedrucktes existierend - nur sehr wenige Bilder bringen konnten, entstanden leicht Stereotypen, eben weil es so wenige und immer die gleichen, also „typische“ Bilder waren. In unserer Gegenwart aber sind Stereotypen lebenskräftiger denn je, obwohl die Bilder so viele sind und durchaus differenziert. Das große Bildermedium Fernsehen umflutet uns mit lauter individuellen Menschen- und Länderbildern, und dennoch bleiben wir gern bei unseren Stereotypen, differenzieren nur widerwillig, freuen uns, wenn wir Stereotypen bestätigt finden. Warum ist das so? Ehe wir eine Antwort zu finden suchen, müssen wir der Frage nähertreten:

\section{Was sind Stereotypen?}

Stereotypen beziehen sich in der Regel auf Menschengruppen, die durch nationale und/oder soziale Merkmale als hinreichend gekennzeichnet gelten. Soziale Stereotypen (festgemacht an Rollen) sind alt, und auch die nationalen Stereotypen sind älter als ihre aggressiven Ausprägungen, die ihnen die Nationalismen des 19. und die totalitären Systeme des 20. Jahrhunderts verschafft haben: Die Engländer sind perfide, die Deutschen spießen kleine Kinder mit dem Bajonett auf, und - man beachte den jeden Zweifel ausschließenden Singular - der Russe macht keine Gefangenen. Während soziale Stereotypen offenbar schon von kleinen Kindern in der Familie erlernt werden („Erziehung durch schlichte Aussagesätze“, sagte Peter R. Hofstätter), kann die Ausbreitung der Nationalismen und ihrer Bilder nicht ohne Mitwirkung der Medien gedacht werden. Es war das gleiche 19. Jahrhundert, in dem auch einfache Menschen in großer Zahl - als „Massenpublikum“ - die Welt als reale Ferne kennen und in dem Völker sich als Nationen im Verhältnis zu angrenzenden, weil abgegrenzten, Nationen sehen lernten. Walter Lippmann, der 1922 als erster die Rolle der Stereotypen in der öffentlichen Meinung analysiert hat, sieht den Menschen als ein Geschöpf, „das gerade eine Portion Wirklichkeit erfassen kann, um sein Leben zu sichern“. Er lernt jetzt, „mit seinem Geist riesige Teile der Welt zu sehen, die er nie zuvor sehen, berühren, riechen, hören oder im Gedächtnis behalten konnte". So entsteht "in seinem Kopf ein Bild von der Welt außerhalb seiner Reichweite ${ }^{\prime 4}$. 
Heute nennen wir das Medienrealität und denken sofort ans Fernsehen. Aber die ersten medienvermittelten „Bilder im Kopf", und zwar auch im Kopf des einfachen Bürgers, sind viel älter. Mit den „Pfennig-Magazinen“ der 1830er Jahre ${ }^{5}$ kamen die wissenschaftlich gemeinten Abbildungen von Elefanten und asiatischen Tempeln oder ägyptischen Pyramiden in jedermanns Haus, und die Zeichner der "illustrierten Zeitungen" lieferten wenig später penible Vorlagen von den Barrikaden der Revolution von 1848 wie von den Kämpfen auf der Krim, der Erstürmung der Düppeler Schanzen und der Schlacht von Königgrätz.

\section{Karikaturen}

Der Siegeszug der Presse-Illustration war zugleich der Siegeszug der massenhaft verbreiteten Karikatur. Und in keiner publizistischen Ausdrucksform lassen sich die "festen Bilder" trefflicher konzentrieren als in der Karikatur. Manche von ihnen sind sehr langlebig. Die preußische Pickelhaube, neben Knobelbecher und Offiziersstiefel das fast zum Symbol verdichtete Piktogramm des Stereotyps vom militaristisch-aggressiven Deutschen, stammt nicht, wie man glauben möchte, aus der alliierten Kriegspropaganda des ersten Weltkriegs, sondern begegnet schon 1848, 1864, 1866 und selbstverständlich 1870/ $71^{6}$. Die deutsche militaristische Selbstbild-Findung ließ 1940 den Knobelbecher von Sieg zu Sieg marschieren, während „Kamerad Schnürschuh“, der österreichische Bundesgenosse und - einen Krieg später - integrierte Wehrmachtssoldat, 42 Jahre nach dem Ende des zweiten Krieges wiederum von preußischer Fußbekleidung eingeholt wird: Ein Foto des Oberleutnants Kurt Waldheim in den typischen Offiziersstiefeln ging um die Welt, ließ in der Anti-WaldheimKampagne anläßlich der Wahl des ehemaligen UNO-Generalsekretärs zum österreichischen Bundespräsidenten alte Feindbilder aufleben und neue entstehen.

Der Hinweis auf die sterotypisierende Potenz der Karikatur soll nicht auf Seitenwege geführt, sondern, wie es dem Bilde zusteht, das Thema anschaulich gemacht haben. Denn auch die Sprache des Nationalismus und der ihm in nicht wenigen Ländern folgenden totalitären Systeme war, wo sie öffentlich wirken wollte, Karikatur. Dokumentarfilme von Hitler- oder Goebbels-Reden lösen bei Studenten der 80er Jahre Gelächter aus. 1940 aber, im Siegesrausch des Mai, dürften Millionen gerührte Freudentränen vor den Volksempfängern geflossen sein. Neville Chamberlain, der friedenswillige britische Premierminister der späten dreißiger Jahre, war für den zehnjährigen Pimpf M.S. selbstverständlich ein Feigling, denn „ein Mann trägt keinen Regenschirm“, und Churchill, stets dicklich und mit Zigarre präsentiert, war sichtlich nicht "hart wie Kruppstahl“, wie ein Hitlerjunge selbstverständlich zu sein hatte.

\section{Warum sind uns Stereotypen willkommen und warum halten wir wider besseres Wissen an ihnen fest?}

Stereotypen sind Verständigungsmittel und Verteidigungsmittel. Man lasse sich nicht täuschen: Verständigungsmittel, das ist nicht dasselbe wie ein Mittel zum Versteben! 
Als Verständigungsmittel erlauben sie einen relativ mühelosen Austausch von Meinungen und Standpunkten, und zwar besonders dort, wo es um heikle Themen geht. Wo ich selbst in einen Verkehrsunfall verwickelt bin, ist es mir nicht unangenehm, wenn der Unfallgegner ein Ausländer ist. Wie der Liedermacher Wolfgang Ambros es einst besungen hat, konnten sich alle Betrachter eines offensichtlichen Mordopfers rasch über den Täter einigen: „Der Hofer war's.“ „Der Hofer" aber war, wie sich in der letzten Strophe herausstellt, das Opfer?.

Hier sind wir wieder bei der Rolle der Medien: Medienbotschaften für den Tag, die Woche, den Monat geschrieben, müssen kurz sein. Wer sie formuliert, muß den Vorrat an Begriffen und Bildern im Kopf seines erhofften Lesers, Hörers, Sehers in Rechnung zu stellen versuchen. Nur wo die Mosaik-Steinchen der Botschaft die vorbereitete Nische im Kopf des Rezipienten treffen, kommt Kommunikation im erhofften Sinngefüge zustande. Will ich das bei sehr vielen Lesern, Hörern, Sehern bewirken, muß ich einen gemeinsamen Nenner finden, der nicht zu hohe Ansprüche stellt. Der Journalist, der sich kurz fassen muß und sein Kommunikationsangebot gelingen lassen will, weiß, daß Stereotypen fast immer in der gemeinsamen Zone der beiderseitigen Verständigungskapazität liegen. Er wird sich ihrer bedienen, wo er es für gefahrlos hält, aber eben auch dort, wo er auf jeden Fall Verständigung bewirken will. Dagegen läßt sich einwenden: Was für den Journalisten ein „Zwang des Mediums“ ist - er muß ja sein Publikum zufriedenstellen, und das in Kürze -, kann den denkenden Einzelnen kalt lassen. Er darf sich den Luxus leisten, differenziert nachzudenken. Ob er das Resultat eines solchen stereotypenfreien Denkprozesses öffentlich preisgeben will?: Oder ob er, wie Noelle-Neumann ${ }^{8}$ es beschreibt, aus Isolationsfurcht schweigt, wenn es um „öffentliche Meinung" geht? Damit sind wir bei der Funktion von Stereotypen als Verteidigungsmittel: Bedient man sich ihrer, ist man normalerweise vor Isolation geschützt, - sicher im eigenem Umfeld. Kommen aber Angriffe von außen gegen die stereotypisierende Argumentationsgemeinschaft, der man sich zugehörig fühlt, so erweisen sich von den Stereotypen besonders die Feindbilder als hilfreich: Wo immer eine Gemeinschaft sich schwächer fühlt, weil nebenan vielleicht wirklich der next-door-giant wohnt, rückt sie zusammen, und wenn dann gar noch einer mit Steinen zu werfen beginnt, und sei es von jenseits des Atlantiks, dann heißt es "Jetzt erst recht!" Sachlich bringt der Stereotypen-Einsatz wenig, emotional viel. "Eine Welt“, sagt Lippmann, ,in der die von uns geachteten Personen sich als unwürdig erweisen und die von uns verachteten als edel, ist nervenzermürbend." Also geben wir nicht gerne zu, „daß es einen Unterschied zwischen unserem und dem Universum gibt".

\section{Medienrealität und Reisebilder}

Diesem „Stereotypenmodell“, das auf Sicherung des von uns Erreichten aus ist, können sich auch Journalisten besten Willens kaum entziehen. Wo immer der Zwang zur Kürze waltet, ordnen sich Elends- und Mißwirtschaftsbilder wie von selbst der Dritten Welt zu, rollen deutsche Fußballspieler wie Panzer das Feld auf. Dennoch meine ich sehen zu können, daß Bilder- und WörterSprache der Journalistik des deutschen Sprachraums zwar nicht "besser" als 
früher, aber doch zunehmend um Differenzierung bemüht ist. Das umfang- und variantenreiche Bildangebot der verschiedenen Fernsehprogramme macht es selbst versimpelnden Printmedien zunehmend unmöglich, Süditalien ausschließlich als Mafia-Land und Schweden als Wohlfahrtsparadies zu stereotypisieren. Hinzu kommt, daß sehr viele Zeitgenossen aus allen Bevölkerungsschichten als Touristen Länder besucht haben, die zu „Pfennig-Magazins“ Zeiten in unerreichbarer Ferne lagen. Auch wenn nur ein Bruchteil dieser Touristen mit offenen Augen und Ohren reist, sollte es inzwischen, d. h. durch Jahre hin akkumuliert, in den Wohlstandsländern Westeuropas einige Millionen Menschen geben, die die Chance hatten, ihr Vorurteil mit der Wirklichkeit an Ort und Stelle zu konfrontieren. Die große Mehrheit reist jedoch offensichtlich nicht mit offenen Augen und sachbezogener Neugier durch die Welt. Sie sehen die Realität, bevorzugen aber verständlicherweise das für sie Angenehme, also Bilder oder was ihnen nahekommt. Im Alltag aber leben sie, was die „world outside" angeht, in und von der Medienrealität. Sie verweigern keineswegs die Aufnahme von Information. Die Prognosen der Kulturpessimisten haben sich nicht bewahrheitet, wonach die Vermehrung des Fernsehangebots zu Lasten der Nutzung des Informationsangebots gehen werde. Vielmehr bleibt ein harter Kern von Informationsnutzung in ungefähr gleichbleibender Zeitmenge erhalten. Dieses Verhalten konzentriert sich jedoch auf harte Informationen, also auf die Tagesschau und die wichtigsten Report- und Dokumentationsmagazine. Es gibt anscheinend eine Art "Existenzminimum" an täglicher Informationsaufnahme.

\section{Information und Unterhaltung}

„Weiche“ Information hat einen schweren Stand gegenüber den ständig wachsenden Unterhaltungsauswahlmöglichkeiten. Konkret: Je mehr Fernsehkanäle erreichbar werden, desto leichter fällt es dem Publikum, Unterhaltendes zu bevorzugen. Nun gebe man sich nicht der Täuschung hin, Unterhaltung bewirke keine Information. Es ist zwar nicht die Absicht von Unterhaltungsmachern, Information zu vermitteln, - „funktional“ aber werden sehr viele Bestandteile von Unterhaltungsangeboten als Information aufgenommen. Umgekehrt werden Informationssendungen funktional oft wie Unterhaltung rezipiert. Gefördert wird dieser Prozeß durch die konkurrenzbedingten Anstrengungen der Rundfunkanstalten, Informationssendungen formal wie Unterhaltungssendungen zu gestalten: „Infotainment“. Diese Strategie gibt es keineswegs nur beim Fernsehen. Zeitschriften aller Art treiben großen technischen Aufwand, um sich dem Käufer- und erhofften Leserpublikum immer "gefälliger" darzubieten (Vielfarbdruck, gutes Papier etc.). Es geht um Akzeptanz und nicht mehr, wie im aufgeklärten Absolutismus, um „Intelligen $z^{\prime \prime}$.

\section{Informationsfülle und Stereotypen}

Die Möglichkeiten, Information durch Augenschein oder "per medium“ zu gewinnen, sind so groß und so vielfältig wie nie zuvor. Paradoxerweise liegt in eben dieser Größe und Vielfalt auch das größte Hindernis für eine tiefgehende Quellen-Ausschöpfung. Als Goethe nach Italien reiste, wußte er, was er gesehen hatte und mit heimbrachte. Wir können es nachlesen. Wir können auch auf seinen Spuren durch Italien reisen, aber wir könnten dabei kaum der Versu- 
chung widerstehen, viel schneller zu reisen. Jules Vernes „In 80 Tagen um die Erde" als Reiseprogramm zu verwirklichen, ist seit langem kein Problem mehr. Zu den technisch veränderten Chancen der Autopsie tritt die Tatsache der wesentlich umfassenderen Voraus-Information. Wer heute Goethe oder Jules Verne nachreist, weiß selbst dann, wenn er über die betreffenden Länder wenig gelesen hat, mehr darüber, als seine Vorbild-Reisenden vor ihrer Reise gewußt haben können. Für jeden, der nicht fernseh-abstinent lebt, ist es heute unmöglich, von irgendeinem Land der Erde überhaupt noch nicht gehört oder gesehen zu haben.

Diese aus Vielfalt und Mobilität erwachsende Differenzierung könnte auch eine erhebliche Vorleistung für die „Ent-Feindbilderung" erbringen. Aber parallel zu ihr entwickelt sich auf den gleichen Feldern von Massenkommunikation und -tourismus das quasiautomatische Paradoxon: Die aus der zunehmenden Differenziertheit sich ergebende Überfülle von Informationen zwingt sehr viele Menschen dazu, mit der Kapazität ihres Kopfes haushälterisch umzugehen und also zu einfachen Bildern zurückzukehren. Gedruckte und gesendete Magazine mögen die Probleme der Dritte-Welt-Länder einfühlsam und mit dem Ehrgeiz der Nuancierung vorstellen und uns glaubwürdig vermitteln, daß die Notleidenden in Äthiopien oder am Amazonas oder in Taiwan an je anderen Nöten leiden, - am Ende bleibt für den Alltagsgebrauch das Bild, das Dritte Welt und Elend und Nicht-Bewältigung von Problemen zum handhabbaren Stereotyp koppelt.

Die Vokabel „Welt" spielt bei den Stereotypen unserer Zeit eine große Rolle. Lippmann hat das "Bild von der Welt", wie es sich in unseren Köpfen bildet, ebenso wenig primär geographisch gesehen wie 50 Jahre nach ihm Niklas Luhmann den „Schritt zur Weltgesellschaft“. Deren Komplexität läßt sich, soweit es um Kommunikation geht, nur mit Hilfe der Massenmedien bewältigen, d. h. durch immer neue Selektionsprozesse, die am Ende wieder zu Lippmanns lebensnotwendigen „Bildern in den Köpfen der Menschen“ führen.

Die Selektionsprozesse, die wir uns um der „Denk-Ökonomie“ willen selber antun, können uns selber gewaltig in die Irre führen. Denn unsere Wünsche, „wie die Welt sein sollte“, können uns beim Auswählen genau so in die Irre führen, wie uns die Überfülle des Medienangebots die Selektion und damit die Gewinnung des "wahren Bildes" schwer macht.

\section{Revision von Feindbildern?}

Die 1989 einsetzenden Radikalveränderungen in Osteuropa liefern uns gute Beispiele für notwendige Stereotypen-Revisionen: Insbesondere viele Intellektuelle - jene nämlich, die im Sozialismus eine wünschenswerte Chance für die Weiterentwicklung der menschlichen Gesellschaft sahen und mit Hilfe der ebenfalls von Intellektuellen gelenkten Medien über die Unvollkommenheiten des „real existierenden Sozialismus“ jahrelang hinwegzusehen bereit waren sie stehen noch heute konsterniert vor der wirklichen Wirklichkeit, die jetzt von den sozialismusbetroffenen Menschen selbst aufgedeckt worden ist. Mehr noch: Was etwa ein Vierteljahrhundert als vom westlichen Kapitalismus initiier- 
tes „Feindbild“ gescholten wurde, nämlich die sehr kritische und negative Bewertung der Zustände in Polen, Ungarn, der CSFR und der DDR, erwies sich inzwischen als der Wahrheit wesentlich näherstehend, das heißt: als Nicht-Stereotypen.

Was für die Nähe gilt, geschieht auch in der Ferne: Im Ideal-Staat der studierenden Jugend, in Nicaragua, wurde ein sozialistisches Regiment abgewählt, weil die Menschen nicht länger schlechter leben wollen, als sie eigentlich könnten.

Jahrelang aber haben wir aus den Medien gelernt, daß die Bewohner Nicaraguas unter sandinistischer Herrschaft viel glücklicher gewesen seien als je zuvor.

So führt uns die Fülle der Bilder, aber auch die Fülle der Daten, die wir uns beschaffen können, aus der Fülle heraus leicht in die Irre, und nicht immer sind Journalisten in der Lage, dem Grundsatz ,audiatur et altera pars" in einer Weise Geltung zu verschaffen, daß wir die richtigen Schlüsse daraus ziehen können.

Es wäre falsch, die Schuld daran allein den Medien oder gar den Journalisten zuzuschieben.

Denn Journalisten sind, abgesehen von ihrer spezifischen Berufsrolle, auch Menschen und als solche ganz normal in die sie umgebende Gesellschaft hineingewachsen. „Stereotypen stellen“, sagt Franz Dröge, „ein soziales Erbe (social heritage) dar, das sich aus den Normen, kollektiven Einstellungen, kulturellen Institutionen, Kommunikationsgewohnheiten etc. von Gruppen und $\mathrm{Na}$ tionen bildet. Dieser gruppenbedingte Lernprozeß ist existenznotwendig für die Gesellschaft, damit die neuen Mitglieder durch Sozialisierung der Einstellungen, des Gemeinten und Gewußten in die Gruppe integriert werden und nicht Außenseiterpositionen einnehmen. Diese Lernmechanismen gibt es sowohl für Kinder als Neumitglieder, als auch für neu hinzukommende Erwachsene, etwa Einwanderer"10.

Auch Journalisten haben ihr Stereotypen-Pensum gelernt. Sie stehen in besonderer, beruflich bedingter Weise unter dem Zwang zur Vereinfachung, dem sich alle Menschen unterwerfen müssen, wenn sie die mehrheitlich aus „mittelbaren" Informationen bestehende "Welt“ bewältigen wollen. Dröge spricht von einer „Abhängigkeit von Informationen, die einen großen Teil der Wirklichkeit in die Vorstellung statt in die Erfahrung verlagern, Bilder wecken. Diese Bilder sind ebenso notwendig nicht so komplex wie die Wirklichkeit, sondern vereinfacht und bilden meist Stereotypen. Diese Vereinfachung rührt daher, daß wir nicht alle Informationen besitzen, einmal abgesehen davon, daß wir sie intellektuell gar nicht verarbeiten könnten. Die fehlenden Daten werden daher künstlich gewonnen, entweder dadurch, daß man geglaubte oder gemeinte Inhalte als Wirklichkeit betrachtet oder indem man Teilerfahrungen generalisiert. Diese zugefügten Elemente enthalten aufgrund ihrer irrationalen Ungeprüftheit viele Stereotypen und sind Verallgemeinerungen lückenhaft rezi- 
pierter Daten. Es sind meist geprägte Formeln, die griffig und leicht anzunehmen, die gängig und in aller Munde sind ${ }^{\text {"11}}$.

Journalisten unterliegen einem weiteren Berufszwang, den Elisabeth Noelle-Neumann „Konsonanz" nennt. Für Journalisten ist "in aller Munde" zunächst einmal das, was im Munde der Berufskollegen ist. Der „Karriere eines Themas", wenn sie erst einmal von den Medien vorangetrieben wird, kann sich der einzelne Journalist kaum entziehen, er kann allenfalls eine andere Meinung zum Thema entwickeln.

\section{Aberwissen ersetzt Aberglauben}

Haben wir nun überhaupt eine echte Chance, unser Wissen von der Welt stereotypenfrei zu gestalten?

Die einzige Möglichkeit wäre die, „mittelbare Informationen“ durch Erfahrungen zu ersetzen. Das ist jedoch nur auf kleinen und in der Nähe liegenden Teilgebieten möglich; denn auch das "Erfahren“ ist, wie jede Arbeit, der Ökonomie der Zeit und der finanziellen Mittel unterworfen. Wenn ich etwa über ein Dritte-Welt-Land aus den Medien nur Negatives mitgeteilt bekomme und mir mit der Zeit Zweifel an dieser Stereotypisierung aufkommen, so kann ich dieses Land bereisen, d. h. „erfahren". Wieviel Zeit brauche ich, um umzulernen? Was geschieht mir, wenn ich die Stereotypen durch die Wirklichkeit bestätigt fände?

Im Mittelalter wußten die Menschen wenig über die Welt. Ihre Wahrheit fanden sie im Glauben. Das Unheimliche, was sie reichlich umgab, bewältigten sie mit Hilfe des Aberglaubens. In der Gegenwart vermuten wir unsere Wahrheit in der Wissenschaft. Das im strengen Sinne Ungewußte, d. h. das im technischökonomischen Sinne nicht von jedem selbst Erfahrbare, bewältigen wir mit Hilfe „mittelbarer Informationen“. Sie stecken notwenigerweise (Kürze, Verständigung) voll von Stereotypen. Sie helfen uns, und sie führen uns in Versuchung, uns die Weltbewältigung leicht zu machen. Statt auf Aber-Glauben verlassen wir uns auf "Aber-Wissen"? Nicht ganz und nicht immer, denn im Unterschied zum Aberglauben enthalten unsere Freund- und Feindbilder stets wirklichkeitsentsprechende Mosaik-Steinchen.

Eine Chance, stereotypen-frei zu leben, gibt es m. E. nicht. Aber es gibt eine Chance, stereotypen-bewußt und das heißt: stereotypen-kritisch zu leben. Das hilfe jedenfalls Feindbilder abbauen, und auch in diesem positiven Sinne spielen die Medien eine Rolle. ${ }^{12}$

\section{Anmerkungen:}

1 G. Kreisler: Wozu brauchen wir Ausländer? In: „Wochenpresse“, Wien, vom 13.4 .1990 , S. $49 \mathrm{f}$.

2 IMAS-Umfrage 9063, November 1989.

3 Ein aus der Barockzeit stammender Katalog im Österreichischen Museum für Volkskunde, Wien. 
4 W. Lippmann: Die öffentliche Meinung (= Public Opinion, New York 1922), München 1964, S. 27 f.

5 Das deutsche „Pfennig-Magazin der Gesellschaft zur Verbreitung gemeinnütziger Kenntnisse" wurde ab 1833 von Johannes Jakob Weber nach englischem Vorbild ( ${ }_{n}$ Penny Magazine", 1832) in Leipzig herausgegeben.

6 eine schöne Auswahl von „Pickelhauben“ bietet z. B. der Ausstellungskatalog „Ereigniskarikaturen“, Westfälisches Landesmuseum Münster 11.9.-13.11.1983.

7 Vgl. W. Ambros: Da Hofa, auf der Langspielplatte „Alles andere zählt net mehr...” (Atom, Nr. 500.002, Wien 1972).

8 E. Noelle-Neumann: Die Schweigespirale. Öffentiche Meinung - unsere soziale Haut, Frankfurt/M., Wien, Berlin ${ }^{2} 1982$.

9 "Intelligenzwesen“ und „Intelligenzblätter" der Barockzeit leiten ihre Bezeichnung nicht vom heutigen Begriffsinhalt von "Intelligenz" ab, sondern von der "Einsichtnahme" in wichtige Kundmachungen; die allgemeine Einsichtnahme sollte der Wohlfahrt des Landes dienen und konnte deshalb mit Nachdruck verpflichtend gemacht werden. Vgl. dazu Handbuch der Zeitungswissenschaft, Leipzig 1940 ff., Sp. 1806-1845.

10 F.W. Dröge: Publizistik und Vorurteil, Münster/W. 1967, S. 127.

11 Ebenda, S. 132.

12 Der Beitrag beruht $\mathrm{z}$. T. auf der Mitarbeit des Autors an der Fernsehdiskussionsreihe „Feindbilder. Wie Völker miteinander umgehen" des ORF. Sie wurde vom Landesstudio Salzburg in Zusammenarbeit mit der Universität Salzburg und den „Salzburger Nachrichten" produziert und 1987/89 ausgestrahlt. Ein Dokumentationsband gleichen Titels erschien 1988 im Wiener Verlag Kremayr \& Scheriau.

\section{SUMMARY}

The number of political asylum-seekers from Third World countries and refugees and emigrants from the former Eastern Block has grown sharply in recent years. As a form of resistance against these "foreigners" old images of foes are revived. Foe images are a special form of stereotype. Although stereotypes are mostly adopted in the course of general socialisation, modern mass madia contribute to their dissemination. Thus arises a paradox: in earlier days, when there were only a few media, a limited amount of information and hardly any pictures in the media, stereotypes were transmitted because there was so little information. Today the receiver is dependent on the use of stereotypes, in order to be able to cope with the profusion of information, particularly of television pictures. The media consumer in the modern industrial societies is - through television familiar with almost every country in the world. He was been able to travel extensively and gather experience. Generally though he can only come to terms with the fill of impressions with the help of simplification. In this sense the mass media carry a great responsibility.

\section{RÉSUMÉ}

Le nombre de damndeurs d'asyle du tiers monde de réfugiés et d'émigrants des anciens pays de l'est a fort augmenté ces dernierés années. La peur des étrangers fait revivre les clichés ennemis. Les clichés ennemis sont une forme particulière de stéréotypes. Bien que les stéréotypes ne sont pas une nouveauté et que ceuse-ci sont au cours du procés de socialisation, les mass média modernes contribuent á leurs propagations. Aujourd'hui, sans l'emploi de stéréotypes, le recipient ne comprendrait pas l'enès informations et surtout l'enès d'images provenant de la television. Le recipient de l'époque moderne industrielle connait presque le monde entier grace a la television. Il a aussi in voyages dans des jays f differents et aussi collextionnes des enjerimas. 


\section{RESUMEN}

El número de asilantes procedientes de paises del tercer mundo y refugiados de países del anterior bloque del este ha aumentado mucho en los últimos anos. Como defensa contra los „foraneos" están resurgiendo viejos prejuicios hóstiles. Prejuicios son una forma especial de esterotipos. A pesar de que los estereotipos son en parte viejos y se aprenden en su mayoría en la socialización general, los mass-media contribuyen a su difusión. Se produce una situación paradójica: En tiempos anteriores, cuando existían solamente pocos medios de comunicación, una cantidad limitada de informaciones y apenas imágenes en los medios de comunicación, se retransmitieron estereotipos, porque había tan pocas informaciones. Hoy en día, el recipiente necesita usar estereotipos, porque de otra manera no podría digerir la cantidad imensa de informaciones y sobre todo imágenes televisivas. El recipiente en sociedades modernas de países industrializados conoce casi todos los países del mundo - por la televisión. También pudo viajar a muchos países y adquirir experiencias. Pero en general, solamente puede digerir la cantidad e informaciones simplificándolas. Los mass-media tienen una gran responsabilidad en este contexto. 